Dandan Ding, Meiling Chi and Fuyi Xu (Shandong)

\title{
THE OPTIMAL TIME DECAY RATES FOR INCOMPRESSIBLE VISCOELASTIC FLUIDS IN CRITICAL FRAMEWORK
}

Abstract. We study the Cauchy problem for multi-dimensional incompressible viscoelastic fluids in the whole space. The optimal time decay rates of strong solution constructed by Qian (2010), and Zhang (2011) in $L^{2}$ critical regularity framework are obtained for low frequencies of the data under a suitable additional condition. The proof relies on an application of Fourier analysis to a mixed parabolic-hyperbolic system, and on a refined time-weighted energy functional. As a by-product, time decay rates of $L^{q}-L^{r}$ type are also captured in the critical framework.

1. Introduction and main results. In this paper, we consider the following multi-dimensional $(N \geq 2)$ incompressible viscoelastic flow:

$$
\left\{\begin{array}{l}
\partial_{t} U+v \cdot \nabla U=\nabla v U \\
\partial_{t} v+v \cdot \nabla v+\nabla p=\mu \Delta v+\nabla \cdot\left(U U^{T}\right), \\
\nabla \cdot v=0
\end{array}\right.
$$

with the initial data

$$
\left.U\right|_{t=0}=U_{0},\left.\quad v\right|_{t=0}=v_{0} .
$$

Here $U(x, t)=\left(U^{i j}(x, t)\right)_{N \times N}$ denotes the deformation tensor, $v(x, t)$ is the fluid velocity and $p(x, t)$ is the hydrodynamic pressure. The system (1.1) is one of the basic macroscopic models for viscoelastic flows, which corresponds to the so-called Hookean linear elasticity. For more physical background of this system we refer to [9, 11].

2020 Mathematics Subject Classification: 35B40, 35Q35, 35L60.

Key words and phrases: optimal decay rates, incompressible viscoelastic fluids, Besov spaces.

Received 18 March 2020.

Published online 27 July 2020. 
Let us assume that $U_{0}$ and $v_{0}$ satisfy the constraints

$$
\left\{\begin{array}{l}
\nabla \cdot v_{0}=0, \\
\operatorname{det} U_{0}=1, \\
\nabla \cdot U_{0}^{T}=0, \\
U_{0}^{l k} \nabla_{l} U_{0}^{i j}-U_{0}^{l j} \nabla_{l} U_{0}^{j k}=0 .
\end{array}\right.
$$

The system (1.1) has been extensively studied. In the framework of Sobolev spaces, Chen and Zhang [4] and Lei et al. [12] proved the well-posedness for the Cauchy problem and the periodic problem, respectively. In 2014, Hu and $\mathrm{Wu}$ [10] obtained optimal $L^{2}$ time decay rates for global smooth solutions and their spatial derivatives and established the weak-strong uniqueness property in the class of finite energy weak solutions. In the framework of Besov spaces, Qian [15] and Zhang [16] independently proved the existence and uniqueness of a local solution, which was also shown to exist globally in time provided the initial data is small in certain norms. However, there is no result on time decay rates for global strong solutions constructed in [15, 16].

The main motivation of this paper is to consider this question. Our main ideas are based on the known global well-posedness from [15, 16], an application of Fourier analysis to a linearized parabolic-hyperbolic system, and a refined time-weighted energy functional. In low frequencies, employing decay estimates for the Fourier transform of Green's function for the linearized system, which are similar to the decay behavior of the heat kernel, and combining this with Duhamel's principle, one can obtain the desired time-weighted energy estimates. In high frequencies, employing the Fourier localization technology, the symmetrizer methods and classical nonlinear estimates including product estimates and commutator estimates, we can obtain decay estimates for the high frequencies of $(\nabla H, v)$. Finally, in order to close the energy estimates, we further exploit some decay estimates with gain of regularity for the high frequencies of $\Lambda v$. With the help of these tools, we finally establish time decay estimates for the strong solution to the Cauchy problem 1.1)-(1.2).

Let us first recall a global-in-time well-posedness result for the Cauchy problem (1.1)-1.2), which is very useful for our proof.

Theorem 1.1 (15, 16]). Assume $\left(U_{0}-I, v_{0}\right) \in \dot{B}_{2,1}^{N / 2} \cap \dot{B}_{2,1}^{N / 2-1} \times \dot{B}_{2,1}^{N / 2-1}$ and $\left(U_{0}, v_{0}\right)$ satisfies 1.3. Then there exist constants $c$ and $M$ such that if

$$
\left\|U_{0}-I\right\|_{\dot{B}_{2,1}^{N / 2} \cap \dot{B}_{2,1}^{N / 2-1}}+\left\|v_{0}\right\|_{\dot{B}_{2,1}^{N / 2-1}} \leq c,
$$

then the Cauchy problem 1.1 -1.2 has a unique global solution $(U-I, v)$ such that

$$
X(t) \leq M\left(\left\|U_{0}-I\right\|_{\dot{B}_{2,1}^{N / 2} \cap \dot{B}_{2,1}^{N / 2-1}}+\left\|v_{0}\right\|_{\dot{B}_{2,1}^{N / 2-1}}\right),
$$


where

$$
\begin{aligned}
X(t):= & \|U-I\|_{\widetilde{L}_{t}^{\infty}\left(\dot{B}_{2,1}^{N / 2-1}\right) \cap L_{t}^{1}\left(\dot{B}_{2,1}^{N / 2+1}\right)}^{\ell}+\|U-I\|_{\widetilde{L}_{t}^{\infty}\left(\dot{B}_{2,1}^{N / 2}\right) \cap L_{t}^{1}\left(\dot{B}_{2,1}^{N / 2}\right)}^{h} \\
& +\|v\|_{\widetilde{L}_{t}^{\infty}\left(\dot{B}_{2,1}^{N / 2-1}\right)}+\|v\|_{L_{t}^{1}\left(\dot{B}_{2,1}^{N / 2+1}\right)} .
\end{aligned}
$$

Now we state our main result.

THEOREM 1.2. Suppose the data $\left(U_{0}-I, v_{0}\right)$ satisfy the assumptions of Theorem 1.1. Denote $\langle\tau\rangle:=\sqrt{1+\tau^{2}}$ and $\alpha:=\min (N / 4+2,1 / 2+N / 2-\varepsilon)$ with $\varepsilon>0$ arbitrarily small. There exists a positive constant $c$ such that if in addition

$$
D_{0}:=\left\|\left(U_{0}-I, v_{0}\right)\right\|_{\dot{B}_{2, \infty}^{-N / 2}}^{\ell} \leq c,
$$

then the global solution $(U-I, v)$ given by Theorem 1.1 satisfies, for all $t \geq 0$,

$$
D(t) \leq C\left(D_{0}+\left\|\left(\Lambda\left(U_{0}-I\right), v_{0}\right)\right\|_{\dot{B}_{2,1}^{N / 2-1}}^{h}\right),
$$

where

$$
\begin{aligned}
D(t):= & \sup _{s \in(-N / 2,2]}\left\|\langle\tau\rangle^{N / 4+s / 2}(U-I, v)\right\|_{L_{t}^{\infty}\left(\dot{B}_{2,1}^{s}\right)}^{\ell}+\left\|\langle\tau\rangle^{\alpha}(\nabla U, v)\right\|_{\widetilde{L}_{t}^{\infty}\left(\dot{B}_{2,1}^{N / 2-1}\right)}^{h} \\
& +\|\tau \nabla v\|_{\widetilde{L}_{t}^{\infty}\left(\dot{B}_{2,1}^{N / 2}\right)}^{h}
\end{aligned}
$$

As an application of Theorem 1.2, we can obtain the following $L^{q}-L^{r}$ type decay rates for the Cauchy problem (1.1)- 1.2.

Corollary 1.3. For the solution $(U-I, v)$ constructed in Theorem 1.1 we have

(i) if $-N / 2<s \leq N / 2$, then

$$
\left\|\Lambda^{s}(U-I)\right\|_{L^{2}} \lesssim\langle t\rangle^{-(N / 4+s / 2)}\left(D_{0}+\left\|\left(\Lambda\left(U_{0}-I\right), v_{0}\right)\right\|_{\dot{B}_{2,1}^{N / 2-1}}^{h}\right),
$$

(ii) if $-N / 2<s \leq N / 2-1$, then

$$
\left\|\Lambda^{s} v\right\|_{L^{2}} \lesssim\langle t\rangle^{-(N / 4+s / 2)}\left(D_{0}+\left\|\left(\Lambda\left(U_{0}-I\right), v_{0}\right)\right\|_{\dot{B}_{2,1}^{N / 2-1}}^{h}\right),
$$

where the fractional derivative operator $\Lambda^{\ell}$ is defined by

$$
\Lambda^{\ell} f:=\mathcal{F}^{-1}\left(|\cdot|^{\ell} \mathcal{F} f\right)
$$

Proof. Employing the homogeneous Littlewood-Paley decomposition for $\Lambda^{s} a$, we have

$$
\Lambda^{s}(U-I)=\sum_{q \in \mathbb{Z}} \dot{\Delta}_{q} \Lambda^{s}(U-I) .
$$


Thus

$$
\left\|\Lambda^{s}(U-I)\right\|_{L^{2}} \lesssim \sum_{q \in \mathbb{Z}}\left\|\Delta_{q} \Lambda^{s}(U-I)\right\|_{L^{2}}=\left\|\Lambda^{s}(U-I)\right\|_{\dot{B}_{2,1}^{0}} .
$$

Based on the low-high frequencies decomposition, we may write

$$
\begin{aligned}
\sup _{t \in[0, T]}\langle t\rangle^{N / 4+s / 2}\left\|\Lambda^{s}(U-I)\right\|_{\dot{B}_{2,1}^{0}} & \\
& \lesssim\left\|\langle t\rangle^{N / 4+s / 2} H\right\|_{L_{T}^{\infty}\left(\dot{B}_{2,1}^{s}\right)}^{\ell}+\left\|\langle t\rangle^{N / 4+s / 2}(U-I)\right\|_{L_{T}^{\infty}\left(\dot{B}_{2,1}^{s}\right.}^{h} .
\end{aligned}
$$

By 1.8 and the definition of $D(t)$, we get

$$
\left\|\langle t\rangle^{N / 4+s / 2}(U-I)\right\|_{L_{T}^{\infty}\left(\dot{B}_{2,1}^{s}\right)}^{\ell} \lesssim D_{0}+\left\|\left(\Lambda\left(U_{0}-I\right), v_{0}\right)\right\|_{\dot{B}_{2,1}^{N / 2-1}}^{h} .
$$

If $-N / 2<s \leq N / 2$, then $\alpha \geq N / 4+s / 2$, and we obtain

$$
\left\|\langle t\rangle^{N / 4+s / 2}(U-I)\right\|_{L_{T}^{\infty}\left(\dot{B}_{2,1}^{s}\right)}^{h} \lesssim D_{0}+\left\|\left(\Lambda\left(U_{0}-I\right), v_{0}\right)\right\|_{\dot{B}_{2,1}^{N / 2-1}}^{h} .
$$

Thus,

$$
\left\|\Lambda^{s}(U-I)\right\|_{L^{2}} \lesssim\langle t\rangle^{-(N / 4+s / 2)}\left(D_{0}+\left\|\left(\Lambda\left(U_{0}-I\right), v_{0}\right)\right\|_{\dot{B}_{2,1}^{N / 2-1}}^{h}\right) .
$$

Similarly, we also obtain an estimate of $v$ for which we need the stronger conditions $-N / 2<s \leq N / 2-1$ for the high frequencies.

REMARK 1.4. Compared with [10], we establish the decay rates of the strong solution in the so-called critical Besov spaces in any dimension $N \geq 2$ and not only for $N=3$. From Corollary 1.3 , we obtain the decay rates of the solution when $s=0,1$ and $N=3$, which coincides with the result of [10]. Additionally, the decay index $s$ can take both negative and nonnegative values, rather than only nonnegative integer values, which improves the classical decay results, as in [10].

Notations. We let $C$ be a positive generic constant that may vary at different places, and denote $A \leq C B$ by $A \lesssim B$. We shall also need the notations, for some $k_{0} \in \mathbb{Z}$,

$$
\begin{gathered}
z^{\ell}:=\sum_{j \leq k_{0}} \dot{\Delta}_{j} z, \quad z^{h}:=z-z^{\ell} \\
\|z\|_{\dot{B}_{p, 1}^{s}}^{\ell}:=\sum_{j \leq k_{0}} 2^{j s}\left\|\dot{\Delta}_{j} z\right\|_{L^{p}}, \quad\|z\|_{\dot{B}_{p, 1}^{s}}^{h}:=\sum_{j \geq k_{0}} 2^{j s}\left\|\dot{\Delta}_{j} z\right\|_{L^{p}} .
\end{gathered}
$$

Noting the small overlap between low and high frequencies, we have

$$
\left\|z^{\ell}\right\|_{\dot{B}_{p, 1}^{s}} \lesssim\|z\|_{\dot{B}_{p, 1}^{s}}^{\ell} \quad \text { and }\left\|z^{h}\right\|_{\dot{B}_{p, 1}^{s}} \lesssim\|z\|_{\dot{B}_{p, 1}^{s}}^{h} \text {. }
$$

2. Littlewood-Paley theory and some useful lemmas. In this section, we introduce some common notations and basic theorems about the Littlewood-Paley decomposition, and present some auxiliary lemmas. 
Let $\mathcal{S}\left(\mathbb{R}^{N}\right)$ be the Schwartz class of rapidly decreasing function. Given $f \in \mathcal{S}\left(\mathbb{R}^{N}\right)$, its Fourier transform $\mathcal{F} f=\widehat{f}$ is defined by

$$
\widehat{f}(\xi)=\int_{\mathbb{R}^{N}} e^{-i x \cdot \xi} f(x) d x .
$$

Let $(\chi, \varphi)$ be a couple of smooth functions valued in $[0,1]$ such that $\chi$ is supported in the ball $\left\{\xi \in \mathbb{R}^{N}:|\xi| \leq 4 / 3\right\}, \varphi$ is supported in the shell $\left\{\xi \in \mathbb{R}^{N}: 3 / 4 \leq|\xi| \leq 8 / 3\right\}, \varphi(\xi):=\chi(\xi / 2)-\chi(\xi)$ and

$$
\begin{array}{ll}
\chi(\xi)+\sum_{j \geq 0} \varphi\left(2^{-j} \xi\right)=1, & \forall \xi \in \mathbb{R}^{N}, \\
\sum_{j \in \mathbb{Z}} \varphi\left(2^{-j} \xi\right)=1, & \forall \xi \in \mathbb{R}^{N} \backslash\{0\} .
\end{array}
$$

The homogeneous frequency localization operators $\dot{\Delta}_{j}$ and $\dot{S}_{j}$ are defined by

$$
\dot{\Delta}_{j} f:=\mathcal{F}^{-1}\left(\varphi\left(2^{-j} \cdot\right) \mathcal{F} f\right), \quad \dot{S}_{j} f:=\sum_{q \leq j-1} \dot{\Delta}_{q} f \quad \text { for } j \in \mathbb{Z} .
$$

We denote by $\mathcal{S}_{h}^{\prime}\left(\mathbb{R}^{N}\right)$ the dual space of $\mathcal{Z}\left(\mathbb{R}^{N}\right)=\left\{f \in \mathcal{S}\left(\mathbb{R}^{N}\right): D^{\alpha} \widehat{f}(0)=0\right.$, where $\alpha$ is any multi-index $\}$; it can also be identified with the quotient space $\mathcal{S}^{\prime}\left(\mathbb{R}^{N}\right) / \mathbb{P}$ with $\mathbb{P}$ the polynomial space. The formal equality

$$
f=\sum_{j \in \mathbb{Z}} \dot{\Delta}_{j} f
$$

holds true for $f \in \mathcal{S}_{h}^{\prime}\left(\mathbb{R}^{N}\right)$ and is called the homogeneous Littlewood-Paley decomposition. One easily verifies that with our choice of $\varphi$,

$$
\dot{\Delta}_{j} \dot{\Delta}_{q} f \equiv 0 \quad \text { if }|j-q| \geq 2 \quad \text { and } \quad \dot{\Delta}_{j}\left(\dot{S}_{q-1} f \dot{\Delta}_{q} f\right) \equiv 0 \quad \text { if }|j-q| \geq 5 .
$$

Let us recall the definition of homogeneous Besov spaces and some of their properties (see [1, 5, 7, 8]).

Definition 2.1. Let $\mathcal{S}^{\prime}$ be the space of all tempered distributions. For $s \in \mathbb{R}$ and $1 \leq p \leq \infty$, we define the homogeneous Besov space $\dot{B}_{p, 1}^{s}$ to be

$$
\dot{B}_{p, 1}^{s}=\left\{f \in \mathcal{S}_{h}^{\prime}:\|f\|_{\dot{B}_{p, 1}^{s}}<\infty\right\}
$$

with

$$
\mathcal{S}_{h}^{\prime}=\left\{f \in \mathcal{S}^{\prime}: \sum_{j \in \mathbb{Z}} \dot{\Delta}_{j} f=f \in \mathcal{S}^{\prime}\right\} \quad \text { and } \quad\|f\|_{\dot{B}_{p, 1}^{s}}=\sum_{j \in \mathbb{Z}} 2^{j s}\left\|\dot{\Delta}_{j} f\right\|_{L^{p}} .
$$

Definition 2.2. Let $s \in \mathbb{R}, 1 \leq p, \rho, r \leq \infty, 0<T \leq \infty$. The homogeneous space-time Besov space $\tilde{L}_{T}^{\rho} \dot{B}_{p, r}^{s}$ is defined by

where

$$
\tilde{L}_{T}^{\rho} \dot{B}_{p, r}^{s}=\left\{f \in \mathbb{R}_{+} \times \mathcal{S}^{\prime}\left(\mathbb{R}^{N}\right):\|f\|_{\tilde{L}_{T}^{\rho} \dot{B}_{p, r}^{s}}<\infty\right\},
$$

$$
\|f\|_{\tilde{L}_{T}^{\rho} \dot{B}_{p, r}^{s}}:=\left\|2^{j s}\right\| \dot{\Delta}_{j} f(t)\left\|_{L^{\rho}\left(0, T ; L^{p}\right)}\right\|_{\ell^{r}}
$$


Obviously, $\tilde{L}_{T}^{1} \dot{B}_{p, 1}^{s}=L_{T}^{1} \dot{B}_{p, 1}^{s}$. By a direct application of Minkowski's inequality, we have the following relations between these spaces:

$$
\begin{gathered}
L_{T}^{\rho} \dot{B}_{p, r}^{s} \hookrightarrow \tilde{L}_{T}^{\rho} \dot{B}_{p, r}^{s}, \quad r \geq \rho, \\
\tilde{L}_{T}^{\rho} \dot{B}_{p, r}^{s} \hookrightarrow L_{T}^{\rho} \dot{B}_{p, r}^{s}, \quad \rho \geq r .
\end{gathered}
$$

The following Bernstein lemma will be frequently used.

Lemma 2.3 ([2]). Let $1 \leq p \leq q \leq \infty$. Assume that $f \in L^{p}\left(\mathbb{R}^{N}\right)$. Then for any $\gamma \in(\mathbb{N} \cup\{0\})^{N}$, there exist constants $C_{1}, C_{2}$ independent of $f, j$ such that

$$
\begin{aligned}
& \operatorname{supp} \widehat{f} \subseteq\left\{|\xi| \leq A_{0} 2^{j}\right\} \Longrightarrow\left\|\partial^{\gamma} f\right\|_{q} \leq C_{1} 2^{j|\gamma|+j N(1 / p-1 / q)}\|f\|_{p}, \\
& \operatorname{supp} \widehat{f} \subseteq\left\{A_{1} 2^{j} \leq|\xi| \leq A_{2} 2^{j}\right\} \Longrightarrow\|f\|_{p} \leq C_{2} 2^{-j|\gamma|} \sup _{|\beta|=|\gamma|}\left\|\partial^{\beta} f\right\|_{p} .
\end{aligned}
$$

We have the following properties of the product in Besov spaces.

Lemma 2.4 ([7, 8]). For all $1 \leq r, p, p_{1}, p_{2} \leq \infty$, there exists a universal positive constant $C$ such that

$$
\begin{aligned}
& \|f g\|_{\dot{B}_{p, r}^{s}} \leq C\|f\|_{L^{\infty}}\|g\|_{\dot{B}_{p, r}^{s}}+C\|g\|_{L^{\infty}}\|f\|_{\dot{B}_{p, r}^{s}} \quad \text { if } s>0 \\
& \|f g\|_{\dot{B}_{p, r}^{s_{1}+s_{2}-N / p}} \leq C\|f\|_{\dot{B}_{p, r}^{s_{1}}}\|g\|_{\dot{B}_{p, \infty}^{s_{2}}} \quad \text { if } s_{1}, s_{2}<N / p \text { and } \\
& s_{1}+s_{2}+N \min \{0,1-2 / p\}>0 \\
& \|f g\|_{\dot{B}_{p, r}^{s}} \leq C\|f\|_{\dot{B}_{p, r}^{s}}\|g\|_{\dot{B}_{p, \infty}^{N / p} \cap L^{\infty}} \quad \text { if }|s|<N / p ; \\
& \|f g\|_{\dot{B}_{2,1}^{s_{1}+s_{2}-N / 2}} \leq C\|f\|_{\dot{B}_{2,1}^{s_{1}}}\|g\|_{\dot{B}_{2,1}^{s_{2}}} \quad \text { if } s_{1}, s_{2} \leq N / 2 \text { and } s_{1}+s_{2}>0 \text {. }
\end{aligned}
$$

Some embedding properties and interpolation inequalities for Besov spaces are given in

\section{LEMMA 2.5 .}

- For any $p \in[1, \infty]$ we have the continuous embedding

$$
\dot{B}_{p, 1}^{0} \hookrightarrow L^{p} \hookrightarrow \dot{B}_{p, \infty}^{0} .
$$

- If $s \in \mathbb{R}, 1 \leq p_{1} \leq p_{2} \leq \infty$ and $1 \leq r_{1} \leq r_{2} \leq \infty$, then

$$
\dot{B}_{p_{1}, r_{1}}^{s} \hookrightarrow \dot{B}_{p_{2}, r_{2}}^{s-d\left(1 / p_{1}-1 / p_{2}\right)} .
$$

- The space $\dot{B}_{p, 1}^{d / p}$ is continuously embedded in the set of bounded continuous functions (going to 0 at infinity if $p<\infty$ ).

- If $1 \leq p, r_{1}, r_{2}, r \leq \infty, \sigma_{1} \neq \sigma_{2}$ and $\theta \in(0,1)$, then

$$
\|f\|_{\dot{B}_{p, r}^{\theta \sigma_{2}+(1-\theta) \sigma_{1}}} \leq C\|f\|_{\dot{B}_{p, r_{1}}^{\sigma_{1}}}^{1-\theta}\|f\|_{\dot{B}_{p, r_{2}}^{\sigma_{2}}}^{\theta} .
$$


Lemma $2.6([8])$. Let $1 \leq p, p_{1} \leq \infty$ and $\sigma \in \mathbb{R}$. There exists a constant $C>0$ depending only on $\sigma$ such that for all $q \in \mathbb{Z}$, we have

$$
\begin{gathered}
\left\|\left[v \cdot \nabla, \partial_{\ell} \dot{\Delta}_{q}\right] a\right\|_{L^{p}} \leq C c_{q} 2^{-q(\sigma-1)}\|\nabla v\|_{\dot{B}_{p_{1}, 1}^{N / p_{1}}}\|\nabla a\|_{\dot{B}_{p, 1}^{\sigma-1}} \\
\quad \text { for }-\min \left(N / p_{1}, N / p^{\prime}\right)<\sigma \leq 1+\min \left(N / p_{1}, N / p\right), \\
\left\|\left[v \cdot \nabla, \dot{\Delta}_{q}\right] a\right\|_{L^{p}} \leq C c_{q} 2^{-q \sigma}\|\nabla v\|_{\dot{B}_{p_{1}, \infty}^{N / p_{1}} \cap L^{\infty}}\|a\|_{\dot{B}_{p, 1}^{\sigma}} \\
\text { for }-\min \left(N / p_{1}, N / p^{\prime}\right)<\sigma<1+N / p_{1},
\end{gathered}
$$

where the commutator $[\cdot, \cdot]$ is defined by $[f, g]=f g-g f$ and $\left(c_{q}\right)_{q \in \mathbb{Z}}$ denotes a sequence such that $\sum_{q \in \mathbb{Z}} c_{q} \leq 1$.

Proposition 2.7 ([1]). Assume $\mu>0, \sigma \in \mathbb{R},(p, r) \in[1, \infty]^{2}$ and $1 \leq \rho_{2} \leq \rho_{1} \leq \infty$. Let $u$ satisfy

$$
\left\{\begin{array}{l}
\partial_{t} u-\mu \Delta u=f \\
\left.u\right|_{t=0}=u_{0}
\end{array}\right.
$$

Then for all $T>0$ the following a priori estimate is fulfilled:

$$
\mu^{1 / \rho_{1}}\|u\|_{\tilde{L}_{T}^{\rho_{1}}\left(\dot{B}_{p, r}^{\sigma+2 / \rho_{1}}\right)} \lesssim\left\|u_{0}\right\|_{\dot{B}_{p, r}^{\sigma}}+\mu^{1 / \rho_{2}-1}\|f\|_{\tilde{L}_{T}^{\rho_{2}}\left(\dot{B}_{p, r}^{\sigma-2+2 / \rho_{2}}\right)} .
$$

REMARK 2.8. The solutions to the Lamé system

$$
\left\{\begin{array}{l}
\partial_{t} u-\mathcal{A} u=f \\
\left.u\right|_{t=0}=u_{0}
\end{array}\right.
$$

also fulfill (2.4).

We finish this subsection by recalling an elementary but useful inequality.

Lemma 2.9 ([14]). Let $r_{1}, r_{2}>0$ satisfy $\max \left\{r_{1}, r_{2}\right\}>1$. Then

$$
\int_{0}^{t}(1+t-\tau)^{-r_{1}}(1+\tau)^{-r_{2}} d \tau \leq C\left(r_{1}, r_{2}\right)(1+t)^{-\min \left\{r_{1}, r_{2}\right\}} .
$$

3. Time decay estimates. In this section, we exhibit optimal time decay estimates of strong solutions to the system (1.1) -1.2 for initial data close to a stable equilibrium state in critical regularity framework. We divide the argument into several steps.

STEP 1: Reformulation of the original system 1.1)- 1.2 . With $H=U-I$, the system (1.1) -1.2 can be reformulated as follows:

$$
\left\{\begin{array}{l}
\partial_{t} H-\nabla v=-v \cdot \nabla H+\nabla v H, \\
\partial_{t} v-\mu \Delta v-\nabla \cdot H+\nabla p=-v \cdot \nabla v+\nabla \cdot\left(H H^{T}\right), \\
\nabla \cdot v=0, \\
\left.H\right|_{t=0}=H_{0},\left.\quad v\right|_{t=0}=v_{0} .
\end{array}\right.
$$


As in [15, define $d^{i j}=-\Lambda^{-1} \nabla_{j} v^{i}$; then $v^{i}=\Lambda^{-1} \nabla_{j} d^{i j}$. From 3.1) we can deduce the following system for $\left(H^{i j}, d^{i j}\right)$ :

$$
\left\{\begin{array}{l}
\partial_{t} H^{i j}+\Lambda d^{i j}=F-v \cdot \nabla H^{i j}=: f \\
\partial_{t} d^{i j}-\mu \Delta d^{i j}-\Lambda H^{i j}=G-v \cdot \nabla d^{i j}=: g,
\end{array}\right.
$$

where

$$
\begin{aligned}
F= & \nabla_{k} v^{i} H^{k j} \\
G= & v \cdot \nabla\left(-\Lambda^{-1} \nabla_{j} v^{i}\right)+\Lambda^{-1} \nabla_{j} \mathcal{P}\left(v \cdot \nabla v^{i}-H^{l k} \nabla_{l} H^{i k}\right) \\
& +\Lambda^{-1} \nabla_{k}\left(H^{l j} \nabla_{l} H^{i k}-H^{l k} \nabla_{l} H^{i j}\right) .
\end{aligned}
$$

SteP 2: Bounds for the low frequencies. Denoting by $A(D)$ the semigroup associated to 3.2 we have, for all $q \in \mathbb{Z}$,

$$
\left(\begin{array}{c}
\dot{\Delta}_{q} H^{i j}(t) \\
\dot{\Delta}_{q} d^{i j}(t)
\end{array}\right)=e^{t A(D)}\left(\begin{array}{c}
\dot{\Delta}_{q} H_{0}^{i j} \\
\dot{\Delta}_{q} d_{0}^{i j}
\end{array}\right)+\int_{0}^{t} e^{(t-\tau) A(D)}\left(\begin{array}{c}
\dot{\Delta}_{q} f(\tau) \\
\dot{\Delta}_{q} g(\tau)
\end{array}\right) d \tau .
$$

From an explicit computation of the action of $e^{t A(D)}$ in Fourier variables (see e.g. [13, 3]), we discover that there exist positive constants $c_{0}$ and $C$ depending only on $q_{0}$ and such that

$$
\left|\mathcal{F}\left(e^{t A(D)} W\right)(\xi)\right| \leq C e^{-c_{0} t|\xi|^{2}}|\mathcal{F} W(\xi)| \quad \text { for all }|\xi| \leq 2^{q_{0}} .
$$

Therefore, using Parseval's equality and the definition of $\dot{\Delta}_{q}$ we get, for all $q \leq q_{0}$,

$$
\left\|e^{t A(D)} \dot{\Delta}_{q} W\right\|_{L^{2}} \lesssim e^{-c_{0} 2^{2 q} t}\left\|\dot{\Delta}_{q} W\right\|_{L^{2}} .
$$

Hence, multiplying by $t^{N / 4+s / 2} 2^{q s}$ and summing over $q \leq q_{0}$ yields

$$
\begin{aligned}
t^{N / 4+s / 2} \sum_{q \leq q_{0}} 2^{q s}\left\|e^{t A(D)} \dot{\Delta}_{q} W\right\|_{L^{2}} & \lesssim \sum_{q \leq q_{0}} 2^{q s} e^{-c_{0} 2^{2 q} t}\left\|\dot{\Delta}_{q} W\right\|_{L^{2}} t^{N / 4+s / 2} \\
& \lesssim \sum_{q \leq q_{0}} 2^{q(s+N / 2)} e^{-c_{0} 2^{2 q} t}\left\|\dot{\Delta}_{q} W\right\|_{L^{2}} 2^{q(-N / 2)} t^{N / 4+s / 2} \\
& \lesssim\|W\|_{\dot{B}_{2, \infty}^{-N / 2}}^{\ell} \sum_{q \leq q_{0}} 2^{q(s+N / 2)} e^{-c_{0} 2^{2 q} t} t^{\frac{1}{2}(s+N / 2)} .
\end{aligned}
$$

For any $\sigma>0$ there exists a constant $C_{\sigma}$ such that

$$
\sup _{t \geq 0} \sum_{q \in \mathbb{Z}} t^{\sigma / 2} 2^{q \sigma} e^{-c_{0} 2^{2 q} t} \leq C_{\sigma} .
$$

We infer from (3.4) and 3.5 that for $s>-N / 2$,

$$
\sup _{t \geq 0} t^{N / 4+s / 2}\left\|e^{t A(D)} W\right\|_{\dot{B}_{2,1}^{s}}^{\ell} \lesssim\|W\|_{\dot{B}_{2, \infty}^{-N / 2}}^{\ell}
$$


It is also obvious that for $s>-N / 2$,

$$
\left\|e^{t A(D)} W\right\|_{\dot{B}_{2,1}^{s}}^{\ell} \lesssim\|W\|_{\dot{B}_{2, \infty}^{-N / 2}}^{\ell} \sum_{q \leq q_{0}} 2^{q(s+N / 2)} \lesssim\|W\|_{\dot{B}_{2, \infty}^{-N / 2}}^{\ell} .
$$

So, setting $\langle t\rangle:=\sqrt{1+t^{2}}$, we arrive at

$$
\sup _{t \geq 0}\langle t\rangle^{N / 4+s / 2}\left\|e^{t A(D)} W\right\|_{\dot{B}_{2,1}^{s}}^{\ell} \lesssim\|W\|_{\dot{B}_{2, \infty}^{-N / 2}}^{\ell},
$$

and thus, taking advantage of Duhamel's formula, we get

$$
\begin{aligned}
\| \int_{0}^{t} e^{(t-\tau) A(D)}(f, g)(\tau) d \tau & \|_{\dot{B}_{2,1}^{s}}^{\ell} \\
& \lesssim \int_{0}^{t}\langle t-\tau\rangle^{-(N / 4+s / 2)}\|(f, g)(\tau)\|_{\dot{B}_{2, \infty}^{-N / 2}}^{\ell} d \tau
\end{aligned}
$$

We claim that for all $s \in(-N / 2,2]$ and all $t \geq 0$,

$$
\int_{0}^{t}\langle t-\tau\rangle^{-(N / 4+s / 2)}\|(f, g)(\tau)\|_{\dot{B}_{2, \infty}^{-N / 2}}^{\ell} d \tau \lesssim\langle t\rangle^{-(N / 4+s / 2)}\left(X(t)^{2}+D(t)^{2}\right)
$$

Owing to the embedding $L^{1} \hookrightarrow \dot{B}_{2, \infty}^{-N / 2}$, it suffices to prove 3.8 with $\|(f, g)(\tau)\|_{L^{1}}^{\ell}$ instead of $\|(f, g)(\tau)\|_{\dot{B}_{2, \infty}^{-N / 2}}^{\ell}$.

To bound the term with $f$, we use the decomposition

$$
f=-v \cdot \nabla H^{i j}+H^{k j}\left(\nabla_{k} v^{i}\right)^{\ell}+H^{k j}\left(\nabla_{k} v^{i}\right)^{h} .
$$

Now, from Hölder's inequality, the embedding $\dot{B}_{2,1}^{0} \hookrightarrow L^{2}$, the definitions of $D(t), \alpha$ and Lemma 2.9. one may write, for all $s \in(-N / 2,2]$,

$$
\begin{aligned}
& \int_{0}^{t}\langle t-\tau\rangle^{-(N / 4+s / 2)}\left\|\left(v \cdot \nabla H^{i j}\right)(\tau)\right\|_{L^{1}}^{\ell} d \tau \\
\lesssim & \int_{0}^{t}\langle t-\tau\rangle^{-(N / 4+s / 2)}\|v\|_{L^{2}}\left\|\nabla H^{i j}\right\|_{L^{2}} d \tau \\
\lesssim & \int_{0}^{t}\langle t-\tau\rangle^{-(N / 4+s / 2)}\|v\|_{\dot{B}_{2,1}^{0}}\left\|\nabla H^{i j}\right\|_{\dot{B}_{2,1}^{0}} d \tau \\
\lesssim & \int_{0}^{t}\langle t-\tau\rangle^{-(N / 4+s / 2)}\left(\|v\|_{\dot{B}_{2,1}^{0}}^{\ell}+\|v\|_{\dot{B}_{2,1}^{0}}^{h}\right)\left(\left\|\left.\nabla H^{i j}\right|_{\dot{B}_{2,1}^{0}} ^{\ell}+\right\| \nabla H^{i j} \|_{\dot{B}_{2,1}^{0}}^{h}\right) d \tau
\end{aligned}
$$




$$
\begin{aligned}
& \lesssim\left(\sup _{0 \leq \tau \leq t}\langle\tau\rangle^{N / 4}\|v(\tau)\|_{\dot{B}_{2,1}^{0}}^{\ell}\right)\left(\sup _{0 \leq \tau \leq t}\langle\tau\rangle^{N / 4+1 / 2}\left\|\nabla H^{i j}\right\|_{\dot{B}_{2,1}^{0}}^{\ell}\right) \\
& \times \int_{0}^{t}\langle t-\tau\rangle^{-(N / 4+s / 2)}\langle\tau\rangle^{-(N / 2+1 / 2)} d \tau \\
& +\left(\sup _{0 \leq \tau \leq t}\langle\tau\rangle^{N / 4}\|v(\tau)\|_{\dot{B}_{2,1}^{0}}^{\ell}\right)\left(\sup _{0 \leq \tau \leq t}\langle\tau\rangle^{\alpha}\left\|\nabla H^{i j}(\tau)\right\|_{\dot{B}_{2,1}^{N / 2-1}}^{h}\right) \\
& \times \int_{0}^{t}\langle t-\tau\rangle^{-(N / 4+s / 2)}\langle\tau\rangle^{-(N / 4+\alpha)} d \tau \\
& +\left(\sup _{0 \leq \tau \leq t}\langle\tau\rangle^{\alpha}\|v(\tau)\|_{\dot{B}_{2,1}^{N / 2-1}}^{h}\right)\left(\sup _{0 \leq \tau \leq t}\langle\tau\rangle^{N / 4+1 / 2}\left\|\nabla H^{i j}\right\|_{\dot{B}_{2,1}^{\ell}}^{\ell}\right) \\
& \times \int_{0}^{t}\langle t-\tau\rangle^{-(N / 4+s / 2)}\langle\tau\rangle^{-(\alpha+N / 4+1 / 2)} d \tau \\
& +\left(\sup _{0 \leq \tau \leq t}\langle\tau\rangle^{\alpha}\|v(\tau)\|_{\dot{B}_{2,1}^{N / 2-1}}^{h}\right)\left(\sup _{0 \leq \tau \leq t}\langle\tau\rangle^{\alpha}\left\|\nabla H^{i j}\right\|_{\dot{B}_{2,1}^{N / 2-1}}^{h}\right) \\
& \times \int_{0}^{t}\langle t-\tau\rangle^{-(N / 4+s / 2)}\langle\tau\rangle^{-2 \alpha} d \tau \\
& \lesssim D(t)^{2} \int_{0}^{t}\langle t-\tau\rangle^{-(N / 4+s / 2)}\langle\tau\rangle^{-\min (N / 2+1 / 2, \alpha+N / 4,2 \alpha)} d \tau \\
& \lesssim\langle t\rangle^{-(N / 4+s / 2)} D(t)^{2}
\end{aligned}
$$

The term $H^{k j}\left(\nabla_{k} v^{i}\right)^{\ell}$ may be treated along the same lines:

$$
\begin{aligned}
& \int_{0}^{t}\langle t-\tau\rangle^{-(N / 4+s / 2)}\left\|H^{k j}\left(\nabla_{k} v^{i}\right)^{\ell}\right\|_{L^{1}} d \tau \\
& \lesssim \int_{0}^{t}\langle t-\tau\rangle^{-(N / 4+s / 2)}\left(\|H\|_{\dot{B}_{2,1}^{0}}^{\ell}+\|H\|_{\dot{B}_{2,1}^{0}}^{h}\right)\|\nabla v\|_{\dot{B}_{2,1}^{0}}^{\ell} d \tau \\
& \lesssim\left(\sup _{0 \leq \tau \leq t}\langle\tau\rangle^{N / 4}\|H(\tau)\|_{\dot{B}_{2,1}^{0}}^{\ell}\right)\left(\sup _{0 \leq \tau \leq t}\langle\tau\rangle^{N / 4+1 / 2}\|v(\tau)\|_{\dot{B}_{2,1}^{1}}^{\ell}\right) \\
& \times \int_{0}^{t}\langle t-\tau\rangle^{-(N / 4+s / 2)}\langle\tau\rangle^{-(N / 2+1 / 2)} d \tau \\
& +\left(\sup _{0 \leq \tau \leq t}\langle\tau\rangle^{\alpha}\|H(\tau)\|_{\dot{B}_{2,1}^{N / 2}}^{h}\right)\left(\sup _{0 \leq \tau \leq t}\langle\tau\rangle^{N / 4+1 / 2}\|v(\tau)\|_{\dot{B}_{2,1}^{1}}^{\ell}\right) \\
& \times \int_{0}^{t}\langle t-\tau\rangle^{-(N / 4+s / 4)}\langle\tau\rangle^{-(\alpha+N / 4+1 / 2)} d \tau \\
& \lesssim D(t)^{2} \int_{0}^{t}\langle t-\tau\rangle^{-(N / 4+s / 2)}\langle\tau\rangle^{-\min (N / 2+1 / 2, \alpha+N / 4+1 / 2)} d \tau \\
& \lesssim\langle t\rangle^{-(N / 4+s / 2)} D(t)^{2}
\end{aligned}
$$


Regarding the term with $H^{k j}\left(\nabla_{k} v^{i}\right)^{h}$, we use the fact that for $t \geq 2$,

$$
\begin{aligned}
\int_{0}^{t}\langle t-\tau\rangle^{-(N / 4+s / 2)} \| & H^{k j}\left(\nabla_{k} v^{i}\right)^{h}(\tau) \|_{L^{1}} d \tau \\
& \lesssim \int_{0}^{t}\langle t-\tau\rangle^{-(N / 4+s / 2)}\|H(\tau)\|_{\dot{B}_{2,1}^{0}}\|\nabla v(\tau)\|_{\dot{B}_{2,1}^{0}}^{h} d \tau \\
& \lesssim \int_{0}^{1}\langle t-\tau\rangle^{-(N / 4+s / 2)}\|H(\tau)\|_{\dot{B}_{2,1}^{0}}\|\nabla v(\tau)\|_{\dot{B}_{2,1}^{0}}^{h} d \tau \\
& +\int_{1}^{t}\langle t-\tau\rangle^{-(N / 4+s / 2)}\|H(\tau)\|_{\dot{B}_{2,1}^{0}}\|\nabla v(\tau)\|_{\dot{B}_{2,1}^{0}}^{h} d \tau \\
= & : I_{1}+I_{2} .
\end{aligned}
$$

Recalling the definitions of $X(t)$ and $D(t)$, we obtain

$$
\begin{aligned}
I_{1} & =\int_{0}^{1}\langle t-\tau\rangle^{-(N / 4+s / 2)}\|H(\tau)\|_{\dot{B}_{2,1}^{0}}\|\nabla v(\tau)\|_{\dot{B}_{2,1}^{0}}^{h} d \tau \\
& \lesssim\langle t\rangle^{-(N / 4+s / 2)} \sup _{0 \leq \tau \leq 1}\|H(\tau)\|_{\dot{B}_{2,1}^{0}} \int_{0}^{1}\|\nabla v(\tau)\|_{\dot{B}_{2,1}^{0}}^{h} d \tau \\
& \lesssim\langle t\rangle^{-(N / 4+s / 2)} \sup _{0 \leq \tau \leq 1}\|H(\tau)\|_{\dot{B}_{2,1}^{0}} \int_{0}^{1}\|v(\tau)\|_{\dot{B}_{2,1}^{N / 2+1}}^{h} d \tau \\
& \lesssim\langle t\rangle^{-(N / 4+s / 2)} D(1) X(1)
\end{aligned}
$$

and, using the fact that $\langle\tau\rangle \approx \tau$ when $\tau \geq 1$,

$$
\begin{aligned}
I_{2} & =\int_{1}^{t}\langle t-\tau\rangle^{-(N / 4+s / 2)}\|H(\tau)\|_{\dot{B}_{2,1}^{0}}\|\nabla v(\tau)\|_{\dot{B}_{2,1}^{0}}^{h} d \tau \\
& \lesssim \int_{1}^{t}\langle t-\tau\rangle^{-(N / 4+s / 2)}\left(\|H(\tau)\|_{\dot{B}_{2,1}^{0}}^{\ell}+\|H(\tau)\|_{\dot{B}_{2,1}^{0}}^{h}\right)\|\nabla v(\tau)\|_{\dot{B}_{2,1}^{0}}^{h} d \tau \\
\lesssim & \left(\sup _{1 \leq \tau \leq t}\langle\tau\rangle^{N / 4}\|H(\tau)\|_{\dot{B}_{2,1}^{0}}^{\ell}\right)\left(\sup _{1 \leq \tau \leq t}\|\tau \nabla v(\tau)\|_{\dot{B}_{2,1}^{N / 2}}^{h}\right) \\
& \times \int_{1}^{t}\langle t-\tau\rangle^{-(N / 4+s / 2)}\langle\tau\rangle^{-(N / 4+1)} d \tau \\
& +\left(\sup _{1 \leq \tau \leq t}\langle\tau\rangle^{\alpha}\|H(\tau)\|_{\dot{B}_{2,1}^{N / 2}}^{h}\right)\left(\sup _{1 \leq \tau \leq t}^{t}\|\tau \nabla v(\tau)\|_{\dot{B}_{2,1}^{N / 2}}^{h}\right) \\
& \times \int_{1}^{t}\langle t-\tau\rangle^{-(N / 4+s / 2)}\langle\tau\rangle^{-(\alpha+1)} d \tau
\end{aligned}
$$




$$
\begin{aligned}
& \lesssim D(t)^{2} \int_{1}^{t}\langle t-\tau\rangle^{-(N / 4+s / 2)}\langle\tau\rangle^{-\min (\alpha+1, N / 4+1)} d \tau \\
& \lesssim\langle t\rangle^{-(N / 4+s / 2)} D(t)^{2} .
\end{aligned}
$$

Therefore, for $t \geq 2$, we arrive at

$$
\begin{aligned}
\int_{0}^{t}\langle t-\tau\rangle^{-(N / 4+s / 2)} \| H^{k j}\left(\nabla_{k} v^{i}\right)^{h} & (\tau) \|_{L^{1}} d \tau \\
& \lesssim\langle t\rangle^{-(N / 4+s / 2)}\left(D(t)^{2}+X(t)^{2}\right) .
\end{aligned}
$$

The case $t \leq 2$ is obvious as $\langle t\rangle \approx 1$ and $\langle t-\tau\rangle \approx 1$ for $0 \leq \tau \leq t \leq 2$, and

$$
\begin{aligned}
\int_{0}^{t}\left\|H^{k j}\left(\nabla_{k} v^{i}\right)^{h}\right\|_{L^{1}} d \tau \\
\quad \lesssim\|H\|_{L_{t}^{\infty}\left(L^{2}\right)}\|\nabla v\|_{L_{t}^{1}\left(L^{2}\right)}^{h} \lesssim\|H\|_{L_{t}^{\infty}\left(\dot{B}_{2,1}^{0}\right)}\|\nabla v\|_{L_{t}^{1}\left(\dot{B}_{2,1}^{0}\right)}^{h} \\
\quad \lesssim\|H\|_{L_{t}^{\infty}\left(\dot{B}_{2,1}^{0}\right)}\|v\|_{L_{t}^{1}\left(\dot{B}_{2,1}^{N / 2+1}\right)}^{h} \lesssim X(t) D(t) .
\end{aligned}
$$

From 3.9-3.12, we get

$$
\int_{0}^{t}\langle t-\tau\rangle^{-(N / 4+s / 2)}\|f(\tau)\|_{\dot{B}_{2, \infty}^{-N / 2}}^{\ell} d \tau \lesssim\langle t\rangle^{-(N / 4+s / 2)}\left(X(t)^{2}+D(t)^{2}\right) .
$$

Next, in order to bound the term of (3.8) corresponding to $g$, we use the decomposition

$$
\begin{aligned}
g:= & G-v \cdot \nabla d^{i j} \\
= & -v \cdot \nabla d^{i j}+v \cdot \nabla\left(-\Lambda^{-1} \nabla_{j} v^{i}\right)+\Lambda^{-1} \nabla_{j} \mathcal{P}\left(v \cdot \nabla v^{i}-H^{l k} \nabla_{l} H^{i k}\right) \\
& +\Lambda^{-1} \nabla_{k}\left(H^{l j} \nabla_{l} H^{i k}-H^{l k} \nabla_{l} H^{i j}\right) .
\end{aligned}
$$

Similar to $3.10-3.12$, we have

$$
\int_{0}^{t}\langle t-\tau\rangle^{-(N / 4+s / 2)}\left\|v \cdot\left(\nabla d^{i j}\right)^{\ell}\right\|_{L^{1}} d \tau \lesssim\langle t\rangle^{-(N / 4+s / 2)} D(t)^{2}
$$

and

$$
\int_{0}^{t}\langle t-\tau\rangle^{-(N / 4+s / 2)}\left\|v \cdot\left(\nabla d^{i j}\right)^{h}\right\|_{L^{1}} d \tau \lesssim\langle t\rangle^{-(N / 4+s / 2)}\left(D(t)^{2}+X(t)^{2}\right) .
$$

Similar to $v \cdot \nabla d^{i j}$, we have

$$
\begin{aligned}
\int_{0}^{t}\langle t-\tau\rangle^{-(N / 4+s / 2)} \| v \cdot \nabla\left(-\Lambda^{-1} \nabla_{j} v^{i}\right) & +\Lambda^{-1} \nabla_{j} \mathcal{P} v \cdot \nabla v^{i} \|_{L^{1}} d \tau \\
& \lesssim\langle t\rangle^{-(N / 4+s / 2)}\left(D(t)^{2}+X(t)^{2}\right)
\end{aligned}
$$


For $\Lambda^{-1} \nabla_{j} \mathcal{P} H^{l k} \nabla_{l} H^{i k}+\Lambda^{-1} \nabla_{k}\left(H^{l j} \nabla_{l} H^{i k}-H^{l k} \nabla_{l} H^{i j}\right)$, similar to (3.9), we have

$$
\begin{array}{r}
\int_{0}^{t}\langle t-\tau\rangle^{-(N / 4+s / 2)}\left\|\Lambda^{-1} \nabla_{j} \mathcal{P} H^{l k} \nabla_{l} H^{i k}+\Lambda^{-1} \nabla_{k}\left(H^{l j} \nabla_{l} H^{i k}-H^{l k} \nabla_{l} H^{i j}\right)\right\|_{L^{1}} d \tau \\
\lesssim\langle t\rangle^{-(N / 4+s / 2)} D(t)^{2}
\end{array}
$$

Thus,

$$
\int_{0}^{t}\langle t-\tau\rangle^{-(N / 4+s / 2)}\|g(\tau)\|_{\dot{B}_{2, \infty}^{-N / 2}}^{\ell} d \tau \lesssim\langle t\rangle^{-(N / 4+s / 2)}\left(X(t)^{2}+D(t)^{2}\right),
$$

and, the proof of $(3.8)$ is complete.

Combining (3.6) and (3.8), we conclude that for all $t \geq 0$ and $s \in$ $(-N / 2,2]$

$$
\langle t\rangle^{N / 4+s / 2}\|(H, v)\|_{\dot{B}_{2,1}^{s}}^{\ell} \lesssim D_{0}+X(t)^{2}+D(t)^{2} .
$$

STEP 3: Decay estimates for the high frequencies of $(\nabla H, v)$. Applying the operator $\dot{\Delta}_{q}$ to the system 3.2 , we deduce that $\left(\dot{\Delta}_{q} H, \dot{\Delta}_{q} v\right)$ satisfies

$$
\left\{\begin{array}{l}
\partial_{t} \dot{\Delta}_{q} H^{i j}+\dot{\Delta}_{q} \Lambda d^{i j}=-\dot{\Delta}_{q}\left(v \cdot \nabla H^{i j}\right)+\dot{\Delta}_{q} F \\
\partial_{t} \dot{\Delta}_{q} d^{i j}-\mu \Delta \dot{\Delta}_{q} d^{i j}-\dot{\Delta}_{q} \Lambda H^{i j}=-\dot{\Delta}_{q}\left(v \cdot \nabla d^{i j}\right)+\dot{\Delta}_{q} G .
\end{array}\right.
$$

Taking the $L^{2}$-scalar product of the first equation of (3.14) with $\dot{\Delta}_{q} H^{i j}$ and $\mu^{2} / 2 \Lambda^{2} \dot{\Delta}_{q} H^{i j}$, and the second equation with $\dot{\Delta}_{q} d^{i j}$, we obtain the following five identities:

$$
\begin{aligned}
\frac{1}{2} \frac{d}{d t}\left\|\dot{\Delta}_{q} H^{i j}\right\|_{L^{2}}^{2}+ & \left(\dot{\Delta}_{q} \Lambda d^{i j} \mid \dot{\Delta}_{q} H^{i j}\right) \\
& =-\left(\dot{\Delta}_{q}\left(v \cdot \nabla H^{i j}\right) \mid \dot{\Delta}_{q} H^{i j}\right)+\left(\dot{\Delta}_{q} F \mid \dot{\Delta}_{q} H^{i j}\right) \\
\frac{\mu^{2}}{4} \frac{d}{d t}\left\|\Lambda \dot{\Delta}_{q} H^{i j}\right\|_{L^{2}}^{2} & +\frac{\mu^{2}}{2}\left(\Lambda \dot{\Delta}_{q} d^{i j} \mid \Lambda^{2} \dot{\Delta}_{q} H^{i j}\right) \\
= & -\frac{\mu^{2}}{2}\left(\dot{\Delta}_{q}\left(v \cdot \nabla H^{i j}\right) \mid \Lambda^{2} \dot{\Delta}_{q} H^{i j}\right)+\frac{\mu^{2}}{2}\left(\dot{\Delta}_{q} F \mid \Lambda^{2} \dot{\Delta}_{q} H^{i j}\right)
\end{aligned}
$$

and

$$
\begin{aligned}
\frac{1}{2} \frac{d}{d t}\left\|\dot{\Delta}_{q} d^{i j}\right\|_{L^{2}}^{2}+\mu \| & \Lambda \dot{\Delta}_{q} d^{i j} \|_{L^{2}}^{2}-\left(\Lambda \dot{\Delta}_{q} H^{i j} \mid \dot{\Delta}_{q} d^{i j}\right) \\
& =-\left(\dot{\Delta}_{q}\left(v \cdot \nabla d^{i j}\right) \mid \dot{\Delta}_{q} d^{i j}\right)+\left(\dot{\Delta}_{q} G \mid \dot{\Delta}_{q} d^{i j}\right) .
\end{aligned}
$$

Applying the operation $\Lambda$ to the first equation in (3.14) and taking the $L^{2}$ 
scalar product with $\dot{\Delta}_{q} d^{i j}$, and the second one with $\dot{\Delta}_{q} \Lambda H^{i j}$, we get

$$
\begin{aligned}
& -\frac{\mu}{2} \frac{d}{d t}\left(\dot{\Delta}_{q} \Lambda H^{i j} \mid \dot{\Delta}_{q} d^{i j}\right)-\frac{\mu}{2}\left\|\Lambda \dot{\Delta}_{q} d^{i j}\right\|_{L^{2}}^{2} \\
& -\frac{\mu^{2}}{2}\left(\Lambda \dot{\Delta}_{q} d^{i j} \mid \Lambda^{2} \dot{\Delta}_{q} H^{i j}\right)+\frac{\mu}{2}\left\|\Lambda \dot{\Delta}_{q} H^{i j}\right\|_{L^{2}}^{2} \\
= & -\frac{\mu}{2}\left(\dot{\Delta}_{q} \Lambda F \mid \dot{\Delta}_{q} d^{i j}\right)-\frac{\mu}{2}\left(\dot{\Delta}_{q} G \mid \Lambda \dot{\Delta}_{q} H^{i j}\right)+\frac{\mu}{2}\left(\dot{\Delta}_{q} \Lambda\left(v \cdot \nabla H^{i j}\right) \mid \dot{\Delta}_{q} d^{i j}\right) \\
+ & \frac{\mu}{2}\left(\dot{\Delta}_{q}\left(v \cdot \nabla d^{i j}\right) \mid \Lambda \dot{\Delta}_{q} H^{i j}\right) .
\end{aligned}
$$

Define

$$
\alpha_{q}^{2}=\left\|\dot{\Delta}_{q} H^{i j}\right\|_{L^{2}}^{2}+\frac{\mu^{2}}{2}\left\|\Lambda \dot{\Delta}_{q} H^{i j}\right\|_{L^{2}}^{2}+\left\|\dot{\Delta}_{q} d^{i j}\right\|_{L^{2}}^{2}-\mu\left(\dot{\Delta}_{q} \Lambda H^{i j} \mid \dot{\Delta}_{q} d^{i j}\right) .
$$

Employing Young's inequality, we have

$$
\left|\mu\left(\dot{\Delta}_{q} \Lambda H^{i j} \mid \dot{\Delta}_{q} d^{i j}\right)\right| \leq \frac{\mu^{2}}{2}\left\|\Lambda \dot{\Delta}_{q} H^{i j}\right\|_{L^{2}}^{2}+\frac{1}{2}\left\|\dot{\Delta}_{q} d^{i j}\right\|_{L^{2}}^{2} .
$$

Hence, there exist positive constants $c_{1}$ and $c_{2}$ such that

$$
c_{1} \alpha_{q}^{2} \leq\left\|\dot{\Delta}_{q} H^{i j}\right\|_{L^{2}}^{2}+\left\|\Lambda \dot{\Delta}_{q} H^{i j}\right\|_{L^{2}}^{2}+\left\|\dot{\Delta}_{q} d^{i j}\right\|_{L^{2}}^{2} \leq c_{2} \alpha_{q}^{2} .
$$

Thus,

$$
\alpha_{q} \approx\left\|\Lambda \dot{\Delta}_{q} H^{i j}\right\|_{L^{2}}+\left\|\dot{\Delta}_{q} d^{i j}\right\|_{L^{2}} \quad \text { for } q>q_{0} .
$$

Combined with 3.15-3.18, this yields

$$
\begin{aligned}
& \frac{1}{2} \frac{d}{d t} \alpha_{q}^{2}+\frac{\mu}{2}\left\|\Lambda \dot{\Delta}_{q} d^{i j}\right\|_{L^{2}}^{2}+\frac{\mu}{2}\left\|\Lambda \dot{\Delta}_{q} H^{i j}\right\|_{L^{2}}^{2} \\
= & -\left(\dot{\Delta}_{q}\left(v \cdot \nabla H^{i j}\right) \mid \dot{\Delta}_{q} H^{i j}\right)-\frac{\mu^{2}}{2}\left(\dot{\Delta}_{q}\left(v \cdot \nabla H^{i j}\right) \mid \Lambda^{2} \dot{\Delta}_{q} H^{i j}\right) \\
& -\left(\dot{\Delta}_{q}\left(v \cdot \nabla d^{i j}\right) \mid \dot{\Delta}_{q} d^{i j}\right) \\
& +\frac{\mu}{2}\left(\dot{\Delta}_{q} \Lambda\left(v \cdot \nabla H^{i j}\right) \mid \dot{\Delta}_{q} d^{i j}\right)+\frac{\mu}{2}\left(\dot{\Delta}_{q}\left(v \cdot \nabla d^{i j}\right) \mid \Lambda \dot{\Delta}_{q} H^{i j}\right) \\
& +\left(\dot{\Delta}_{q} F \mid \dot{\Delta}_{q} H^{i j}\right)+\frac{\mu^{2}}{2}\left(\dot{\Delta}_{q} F \mid \Lambda^{2} \dot{\Delta}_{q} H^{i j}\right)+\left(\dot{\Delta}_{q} G \mid \dot{\Delta}_{q} d^{i j}\right) \\
& -\frac{\mu}{2}\left(\dot{\Delta}_{q} \Lambda F \mid \dot{\Delta}_{q} d^{i j}\right)-\frac{\mu}{2}\left(\dot{\Delta}_{q} G \mid \Lambda \dot{\Delta}_{q} H^{i j}\right) \\
\lesssim & \alpha_{q}\left(\left\|\dot{\Delta}_{q}\left(v \cdot \nabla H^{i j}\right)\right\|_{L^{2}}+\left\|\Lambda \dot{\Delta}_{q}\left(v \cdot \nabla H^{i j}\right)\right\|_{L^{2}}+\left\|\dot{\Delta}_{q}\left(v \cdot \nabla d^{i j}\right)\right\|_{L^{2}}\right. \\
& \left.+\left\|\dot{\Delta}_{q} F\right\|_{L^{2}}+\left\|\dot{\Delta}_{q} \Lambda F\right\|_{L^{2}}+\left\|\dot{\Delta}_{q} G\right\|_{L^{2}}\right) .
\end{aligned}
$$


For $q \geq q_{0}$ and for some $c_{0}=c\left(q_{0}\right)>0$, we have

$$
\begin{aligned}
\frac{1}{2} \frac{d}{d t} & \alpha_{q}^{2}+c_{0} \alpha_{q}^{2} \\
\lesssim & \alpha_{q}\left(\left\|\dot{\Delta}_{q}\left(v \cdot \nabla H^{i j}\right)\right\|_{L^{2}}+\left\|\Lambda \dot{\Delta}_{q}\left(v \cdot \nabla H^{i j}\right)\right\|_{L^{2}}+\left\|\dot{\Delta}_{q}\left(v \cdot \nabla d^{i j}\right)\right\|_{L^{2}}\right. \\
& \left.+\left\|\dot{\Delta}_{q} F\right\|_{L^{2}}+\left\|\dot{\Delta}_{q} \Lambda F\right\|_{L^{2}}+\left\|\dot{\Delta}_{q} G\right\|_{L^{2}}\right) \\
\lesssim & \left(\left\|\left(F_{q}, \Lambda F_{q}, G_{q}\right)\right\|_{L^{2}}+\left\|R_{q}\left(v, H^{i j}\right)\right\|_{L^{2}}+\left\|R_{q}\left(v, d^{i j}\right)\right\|_{L^{2}}\right. \\
& \left.+\left\|\widetilde{R}_{k}\left(v, H^{i j}\right)\right\|_{L^{2}}+\|\nabla v\|_{L^{\infty}} \alpha_{q}\right) \alpha_{q},
\end{aligned}
$$

where

$$
F_{q}=\dot{\Delta}_{q} F, \quad G_{q}=\dot{\Delta}_{q} G
$$

with

$$
\begin{aligned}
R_{q}(v, b) & :=\left[v \cdot \nabla, \dot{\Delta}_{q}\right] b=v \cdot \nabla \dot{\Delta}_{q} b-\dot{\Delta}_{q}(v \cdot \nabla b) \quad \text { for } b \in\left\{H^{i j}, b^{i j}\right\}, \\
\widetilde{R}_{q}^{i}\left(v, H^{i j}\right) & :=\left[v \cdot \nabla, \partial_{i} \dot{\Delta}_{q}\right] H^{i j}=v \cdot \nabla \partial_{i} \dot{\Delta}_{q} H^{i j}-\partial_{i} \dot{\Delta}_{q}\left(v \cdot \nabla H^{i j}\right) .
\end{aligned}
$$

After time integration, we discover that

$$
\begin{aligned}
e^{c_{0} t} \alpha_{q}(t) \leq \alpha_{q}(0)+\int_{0}^{t} e^{c_{0} \tau}\left(\left\|\left(F_{q}, \Lambda F_{q}, G_{q}\right)\right\|_{L^{2}}+\left\|R_{q}\left(v, H^{i j}\right)\right\|_{L^{2}}\right. \\
\left.\quad+\left\|R_{q}\left(v, d^{i j}\right)\right\|_{L^{2}}+\left\|\widetilde{R}_{k}\left(v, H^{i j}\right)\right\|_{L^{2}}+\|\nabla v\|_{L^{\infty} \alpha_{q}}\right) d \tau .
\end{aligned}
$$

For $q \geq q_{0}$, we have $\alpha_{q} \approx\left\|\left(\Lambda \dot{\Delta}_{q} H^{i j}, \dot{\Delta}_{q} d^{i j}\right)\right\|_{L^{2}}$. Then

$$
\begin{aligned}
\langle t\rangle^{\alpha} \|( & \left.\Lambda \dot{\Delta}_{q} H^{i j}, \dot{\Delta}_{q} d^{i j}\right)(t)\left\|_{L^{2}} \lesssim\langle t\rangle^{\alpha} e^{-c_{0} t}\right\|\left(\Lambda \dot{\Delta}_{q} H^{i j}, \dot{\Delta}_{q} d^{i j}\right)(0) \|_{L^{2}} \\
& +\langle t\rangle^{\alpha} \int_{0}^{t} e^{c_{0}(\tau-t)}\left(\left\|\left(F_{q}, \Lambda F_{q}, G_{q}\right)\right\|_{L^{2}}+\left\|R_{q}\left(v, H^{i j}\right)\right\|_{L^{2}}+\left\|R_{q}\left(v, d^{i j}\right)\right\|_{L^{2}}\right. \\
& \left.+\left\|\widetilde{R}_{k}\left(v, H^{i j}\right)\right\|_{L^{2}}+\|\nabla v\|_{L^{\infty} \alpha_{q}}\right) d \tau
\end{aligned}
$$

and multiplying both sides by $2^{(N / 2-1) q}$, taking the supremum on $[0, T]$, and summing over $q \geq q_{0}$, we get

$$
\begin{aligned}
& \left\|\langle t\rangle^{\alpha}\left(\Lambda H^{i j}, d^{i j}\right)\right\|_{\widetilde{L}_{T}^{\infty}\left(\dot{B}_{2,1}^{N / 2-1}\right)}^{h} \\
& \quad \lesssim\left\|\left(\Lambda H_{0}^{i j}, d_{0}^{i j}\right)\right\|_{\dot{B}_{2,1}^{N / 2-1}}^{h}+\sum_{q \geq q_{0}} \sup _{0 \leq t \leq T}\langle t\rangle^{\alpha} \int_{0}^{t} e^{c_{0}(\tau-t)} 2^{(N / 2-1) q} S_{q} d \tau
\end{aligned}
$$

with $S_{q}:=\sum_{i=1}^{5} S_{q}^{i}$ and

$$
\begin{array}{ll}
S_{q}^{1}:=\left\|\left(F_{q}, \Lambda F_{q}, G_{q}\right)\right\|_{L^{2}}, & S_{q}^{2}:=\left\|R_{q}\left(v, H^{i j}\right)\right\|_{L^{2}}, \quad S_{q}^{3}:=\left\|R_{q}\left(v, d^{i j}\right)\right\|_{L^{2}}, \\
S_{q}^{4}:=\left\|\widetilde{R}_{k}\left(v, H^{i j}\right)\right\|_{L^{2}}, & S_{q}^{5}:=\|\nabla v\|_{L^{\infty}}\left\|\left(\Lambda \dot{\Delta}_{q} H^{i j}, \dot{\Delta}_{q} d^{i j}\right)\right\|_{L^{2}} .
\end{array}
$$


Bounding the sum, for $0 \leq t \leq 2$, and taking advantage of Proposition 2.6. we end up with

$$
\begin{aligned}
& \sum_{q \geq q_{0}} \sup _{0 \leq t \leq 2}\langle t\rangle^{\alpha} \int_{0}^{t} e^{c_{0}(\tau-t)} 2^{(N / 2-1) q} S_{q}(\tau) d \tau \lesssim \int_{0}^{2} \sum_{q \geq q_{0}} 2^{(N / 2-1) q} S_{q}(\tau) d \tau \\
& \lesssim \int_{0}^{2}\left(\|(F, \Lambda F, G)\|_{\dot{B}_{2,1}^{N / 2-1}}^{h}+\|\nabla v\|_{\dot{B}_{2,1}^{N / 2}}\left\|\left(H^{i j}, d^{i j}, \nabla H^{i j}\right)\right\|_{\dot{B}_{2,1}^{N / 2-1}}\right) d \tau \\
& \lesssim \int_{0}^{2}\left(\|(\Lambda F, G)\|_{\dot{B}_{2,1}^{N / 2-1}}^{h}+\|\nabla v\|_{\dot{B}_{2,1}^{N / 2}}\left\|\left(H^{i j}, d^{i j}, \nabla H^{i j}\right)\right\|_{\dot{B}_{2,1}^{N / 2-1}}\right) d \tau \\
& =: Q_{1}+Q_{2} .
\end{aligned}
$$

From Lemma 2.4, we bound the terms $Q_{1}$ and $Q_{2}$ as follows:

$$
\begin{aligned}
\int_{0}^{2}\|\Lambda F\|_{\dot{B}_{2,1}^{N / 2-1}}^{h} d \tau & \lesssim \int_{0}^{2}\|F\|_{\dot{B}_{2,1}^{N / 2}}^{h} d \tau \lesssim \int_{0}^{2}\left\|\nabla_{k} v^{i} H^{k j}\right\|_{\dot{B}_{2,1}^{N / 2}}^{h} d \tau \\
& \lesssim \int_{0}^{2}\left\|\nabla_{k} v^{i}\right\|_{\dot{B}_{2,1}^{N / 2}}\left\|H^{k j}\right\|_{\dot{B}_{2,1}^{N / 2}} d \tau \\
& \lesssim\left\|H^{k j}\right\|_{L_{t}^{\infty}\left(\dot{B}_{2,1}^{N / 2}\right)}\left\|\nabla_{k} v^{i}\right\|_{L_{t}^{1}\left(\dot{B}_{2,1}^{N / 2}\right)} \lesssim X(2)^{2},
\end{aligned}
$$

$$
\begin{aligned}
\int_{0}^{2}\|G\|_{\dot{B}_{2,1}^{N / 2-1}}^{h} d \tau \\
\quad \lesssim \int_{0}^{2}\left\|v \cdot \nabla\left(-\Lambda^{-1} \nabla_{j} v^{i}\right)+\Lambda^{-1} \nabla_{j} \mathcal{P} v \cdot \nabla v^{i}\right\|_{\dot{B}_{2,1}^{N / 2-1}}^{h} d \tau \\
\quad+\int_{0}^{2}\left\|\Lambda^{-1} \nabla_{j} \mathcal{P} H^{l k} \nabla_{l} H^{i k}+\Lambda^{-1} \nabla_{k}\left(H^{l j} \nabla_{l} H^{i k}-H^{l k} \nabla_{l} H^{i j}\right)\right\|_{\dot{B}_{2,1}^{N / 2-1}}^{h} d \tau \\
\quad \lesssim \int_{0}^{2}\|v\|_{\dot{B}_{2,1}^{N / 2-1}}\|\nabla v\|_{\dot{B}_{2,1}^{N / 2}} d \tau+\int_{0}^{2}\|H\|_{\dot{B}_{2,1}^{N / 2}}\|\nabla H\|_{\dot{B}_{2,1}^{N / 2-1}} d \tau \\
\quad \lesssim\|v\|_{L_{t}^{\infty}\left(\dot{B}_{2,1}^{N / 2-1}\right)}\|\nabla v\|_{L_{t}^{1}\left(\dot{B}_{2,1}^{N / 2}\right)}+\|H\|_{L_{t}^{2}\left(\dot{B}_{2,1}^{N / 2}\right)}\|\nabla H\|_{L_{t}^{2}\left(\dot{B}_{2,1}^{N / 2-1}\right)} \\
\quad \lesssim X(2)^{2},
\end{aligned}
$$

and

$$
\begin{aligned}
\int_{0}^{2}\|\nabla v\|_{\dot{B}_{2,1}^{N / 2}} \| & \left(H^{i j}, d^{i j}, \nabla H^{i j}\right) \|_{\dot{B}_{2,1}^{N / 2-1}} d \tau \\
& \lesssim\|\nabla v\|_{L_{T}^{1}\left(\dot{B}_{2,1}^{N / 2}\right)}\left\|\left(H^{i j}, d^{i j}, \nabla H^{i j}\right)\right\|_{L_{T}^{\infty}\left(\dot{B}_{2,1}^{N / 2-1}\right)} \lesssim X(2)^{2} .
\end{aligned}
$$


Therefore, for $t \leq 2$,

$$
\sum_{q \geq q_{0}} \sup _{0 \leq t \leq 2}\langle t\rangle^{\alpha} \int_{0}^{t} e^{c_{0}(\tau-t)} 2^{(N / 2-1) q} S_{q} d \tau \lesssim X(2)^{2} .
$$

To bound the supremum on $[2, T]$, we split the integral on $[0, t]$ into integrals on $[0,1]$ and $[1, t]$, respectively. The $[0,1]$ part is easy to handle:

$$
\begin{aligned}
\sum_{q \geq q_{0}} \sup _{2 \leq t \leq T}\langle t\rangle^{\alpha} \int_{0}^{1} e^{c_{0}(\tau-t)} 2^{(N / 2-1) q} S_{q} d \tau & \leq \sum_{q \geq q_{0}} \sup _{2 \leq t \leq T}\langle t\rangle^{\alpha} e^{-c_{0} / 2 t} \int_{0}^{1} 2^{(N / 2-1) q} S_{q} d \tau \\
& \lesssim \int_{0}^{1} \sum_{q \geq q_{0}} 2^{(N / 2-1) q} S_{q} d \tau
\end{aligned}
$$

Hence

$$
\sum_{q \geq q_{0}} \sup _{2 \leq t \leq T}\langle t\rangle^{\alpha} \int_{0}^{1} e^{c_{0}(\tau-t)} 2^{(N / 2-1) q} S_{q}(\tau) d \tau \lesssim X(1)^{2} .
$$

Let us finally consider the $[1, t]$ part of the integral for $2 \leq t \leq T$. We shall use repeatedly the inequalities

$$
\|\tau \nabla v\|_{\widetilde{L}_{t}^{\infty}\left(\dot{B}_{2,1}^{N / 2}\right)} \lesssim D(t),
$$

which are straightforward for the high frequencies, while for the low frequencies they stem from

$$
\|\tau \nabla v\|_{\widetilde{L}_{t}^{\infty}\left(\dot{B}_{2,1}^{N / 2}\right)}^{\ell} \lesssim\left\|\langle\tau\rangle^{N / 4+1 / 2} v\right\|_{L_{t}^{\infty}\left(\dot{B}_{2,1}^{1}\right)}^{\ell} \lesssim D(t) .
$$

Regarding the contribution of $S_{q}^{1}$, by Lemma 2.9 we first notice that

$$
\left.\sum_{q \geq q_{0}} \sup _{2 \leq t \leq T}\langle t\rangle^{\alpha} \int_{1}^{t} e^{c_{0}(\tau-t)} 2^{(N / 2-1) q} S_{q}^{1}(\tau) d \tau\right) \lesssim\left\|\tau^{\alpha}(\Lambda F, G)\right\|_{\widetilde{L}_{T}^{\infty}\left(\dot{B}_{2,1}^{N / 2-1}\right)}^{h} .
$$

Now, product laws in tilde spaces ensure that

$$
\left\|\tau^{\alpha} \Lambda F\right\|_{\widetilde{L}_{T}^{\infty}\left(\dot{B}_{2,1}^{N / 2-1}\right)}^{h} \lesssim\left\|\tau^{\alpha-1} H\right\|_{\widetilde{L}_{T}^{\infty}\left(\dot{B}_{2,1}^{N / 2}\right)}\|\tau \nabla v\|_{\widetilde{L}_{T}^{\infty}\left(\dot{B}_{2,1}^{N / 2}\right)} .
$$

The high frequencies of the first term are obviously bounded by $D(T)$ :

$$
\left\|\tau^{\alpha-1} H\right\|_{\widetilde{L}_{T}^{\infty}\left(\dot{B}_{2,1}^{N / 2}\right)}^{h} \lesssim\left\|\tau^{\alpha} H\right\|_{\widetilde{L}_{T}^{\infty}\left(\dot{B}_{2,1}^{N / 2}\right)}^{h} \lesssim D(T) .
$$

As for the low frequencies of the first term, we notice that if $N \leq 4$ then for 
D. Ding et al.

all small enough $\varepsilon>0$,

$$
\begin{aligned}
\left\|\tau^{\alpha-1} H\right\|_{\widetilde{L}_{T}^{\infty}\left(\dot{B}_{2,1}^{N / 2}\right)}^{\ell} & \lesssim\left\|\tau^{\alpha-1} H\right\|_{L_{T}^{\infty}\left(\dot{B}_{2,1}^{N / 2-2 \varepsilon}\right)}^{\ell} \\
& \lesssim\left\|\tau^{\alpha-1-N / 2+\varepsilon} \tau^{N / 2-\varepsilon} H\right\|_{L_{T}^{\infty}\left(\dot{B}_{2,1}^{N / 2-2 \varepsilon}\right)}^{\ell} \\
& \lesssim\left\|\tau^{N / 2-\varepsilon} c\right\|_{L_{T}^{\infty}\left(\dot{B}_{2,1}^{N / 2-2 \varepsilon}\right)}^{\ell} \lesssim D(T),
\end{aligned}
$$

and if $N \geq 5$ then

$$
\begin{aligned}
\left\|\tau^{\alpha-1} H\right\|_{\widetilde{L}_{T}^{\infty}\left(\dot{B}_{2,1}^{N / 2}\right)}^{\ell} & \lesssim\left\|\tau^{\alpha-1} H\right\|_{\widetilde{L}_{T}^{\infty}\left(\dot{B}_{2,1}^{2}\right)}^{\ell} \\
& \lesssim\left\|\tau^{\alpha-2-N / 4} \tau^{N / 4+1} H\right\|_{L_{T}^{\infty}\left(\dot{B}_{2,1}^{2}\right)}^{\ell} \\
& \lesssim\left\|\tau^{N / 4+1} H\right\|_{L_{T}^{\infty}\left(\dot{B}_{2,1}^{2}\right)}^{\ell} \lesssim D(T) .
\end{aligned}
$$

Combining 3.25-3.27, we obtain

$$
\left\|\tau^{\alpha-1} H\right\|_{\widetilde{L}_{T}^{\infty}\left(\dot{B}_{2,1}^{N / 2}\right)} \lesssim D(T) .
$$

Therefore, using 3.24 and 3.28 we get

$$
\left\|\tau^{\alpha} \Lambda F\right\|_{\widetilde{L}_{T}^{\infty}\left(\dot{B}_{2,1}^{N / 2-1}\right)}^{h} \lesssim D(T)^{2} .
$$

Noticing that $G=v \cdot \nabla\left(-\Lambda^{-1} \nabla_{j} v^{i}\right)+\Lambda^{-1} \nabla_{j} \mathcal{P}\left(v \cdot \nabla v^{i}-H^{l k} \nabla_{l} H^{i k}\right)+$ $\Lambda^{-1} \nabla_{k}\left(H^{l j} \nabla_{l} H^{i k}-H^{l k} \nabla_{l} H^{i j}\right)$, similar to 3.28$)$ we get

$$
\left\|\tau^{\alpha-1} v\right\|_{\widetilde{L}_{T}^{\infty}\left(\dot{B}_{2,1}^{N / 2-1}\right)} \lesssim D(T) .
$$

Employing 3.24 and 3.29, we obtain

$$
\begin{aligned}
\| \tau^{\alpha}\left(v \cdot \nabla\left(-\Lambda^{-1} \nabla_{j} v^{i}\right)\right. & \left.+\Lambda^{-1} \nabla_{j} \mathcal{P}\left(v \cdot \nabla v^{i}\right)\right) \|_{\widetilde{L}_{T}^{\infty}\left(\dot{B}_{2,1}^{N / 2-1}\right)} \\
& \lesssim\left\|\tau^{\alpha-1} v\right\|_{\widetilde{L}_{T}^{\infty}\left(\dot{B}_{2,1}^{N / 2-1}\right)}\|\tau \nabla v\|_{\widetilde{L}_{T}^{\infty}\left(\dot{B}_{2,1}^{N / 2}\right)} \lesssim D(T)^{2} .
\end{aligned}
$$

Product laws in tilde spaces ensure that

$$
\begin{aligned}
\left\|\tau^{\alpha}\left(\Lambda^{-1} \nabla_{j} \mathcal{P}\left(H^{l k} \nabla_{l} H^{i k}\right)+\Lambda^{-1} \nabla_{k}\left(H^{l j} \nabla_{l} H^{i k}-H^{l k} \nabla_{l} H^{i j}\right)\right)\right\|_{\widetilde{L}_{T}^{\infty}\left(\dot{B}_{2,1}^{N / 2-1}\right)} \\
\lesssim\left\|\tau^{\alpha / 2} H\right\|_{\widetilde{L}_{T}^{\infty}\left(\dot{B}_{2,1}^{N / 2}\right)}^{2}
\end{aligned}
$$

The high frequencies of the first term are obviously bounded by $D(T)$ :

$$
\left\|\tau^{\alpha / 2} H\right\|_{\widetilde{L}_{T}^{\infty}\left(\dot{B}_{2,1}^{N / 2}\right)}^{h} \lesssim\left\|\tau^{\alpha} H\right\|_{\widetilde{L}_{T}^{\infty}\left(\dot{B}_{2,1}^{N / 2}\right)}^{h} \lesssim D(T)
$$

As for the low frequencies of the first term, we notice that if $N \leq 4$ then for 
all small enough $\varepsilon>0$,

$$
\begin{aligned}
\left\|\tau^{\alpha / 2} H\right\|_{\tilde{L}_{T}^{\infty}\left(\dot{B}_{2,1}^{N / 2}\right)}^{\ell} & \lesssim\left\|\tau^{\alpha / 2} H\right\|_{L_{T}^{\infty}\left(\dot{B}_{2,1}^{N / 2-2 \varepsilon}\right)}^{\ell} \\
& \lesssim\left\|\tau^{\alpha / 2-N / 2+\varepsilon N / 2-\varepsilon} H\right\|_{L_{T}^{\infty}\left(\dot{B}_{2,1}^{N / 2-2 \varepsilon}\right)}^{\ell} \lesssim D(T),
\end{aligned}
$$

and if $N \geq 5$ then

$$
\begin{aligned}
\left\|\tau^{\alpha / 2} H\right\|_{\widetilde{L}_{T}^{\infty}\left(\dot{B}_{2,1}^{N / 2}\right)}^{\ell} & \lesssim\left\|\tau^{\alpha / 2} H\right\|_{\widetilde{L}_{T}^{\infty}\left(\dot{B}_{2,1}^{2}\right)}^{\ell} \\
& \lesssim\left\|\tau^{\alpha / 2-N / 4-1} \tau^{N / 4+1} H\right\|_{L_{T}^{\infty}\left(\dot{B}_{2,1}^{2}\right)}^{\ell} \lesssim D(T) .
\end{aligned}
$$

Combining 3.30-3.32, we obtain

$$
\left\|\tau^{\alpha / 2} H\right\|_{\widetilde{L}_{T}^{\infty}\left(\dot{B}_{2,1}^{N / 2}\right)} \lesssim D(T) .
$$

Therefore,

$$
\begin{aligned}
\left\|\tau^{\alpha}\left(\Lambda^{-1} \nabla_{j} \mathcal{P}\left(H^{l k} \nabla_{l} H^{i k}\right)+\Lambda^{-1} \nabla_{k}\left(H^{l j} \nabla_{l} H^{i k}-H^{l k} \nabla_{l} H^{i j}\right)\right)\right\|_{\widetilde{L}_{T}^{\infty}\left(\dot{B}_{2,1}^{N / 2-1}\right)} & \lesssim D(T)^{2} . \\
& \lesssim D(
\end{aligned}
$$

Thus,

$$
\left\|\tau^{\alpha} G\right\|_{\widetilde{L}_{T}^{\infty}\left(\dot{B}_{2,1}^{N / 2-1}\right)}^{h} \lesssim D(T)^{2}
$$

We end up with

$$
\sum_{q \geq q_{0}} \sup _{2 \leq t \leq T}\langle t\rangle^{\alpha} \int_{1}^{t} e^{c_{0}(\tau-t)} 2^{(N / 2-1) q} S_{q}^{1}(\tau) d \tau \lesssim X(T)^{2}+D(T)^{2} .
$$

To bound the term with $S_{q}^{2}$, we use the fact that

$$
\int_{1}^{t} e^{c_{0}(\tau-t)}\left\|R_{q}\left(v, H^{i j}\right)\right\|_{L^{2}} d \tau \leq\left\|R_{q}\left(\tau v, \tau^{\alpha-1} H^{i j}\right)\right\|_{L_{t}^{\infty}\left(L^{2}\right)} \int_{1}^{t} e^{c_{0}(\tau-t)} \tau^{-\alpha} d \tau .
$$

Hence, thanks to Lemma 2.9 and Proposition 2.6.

$$
\begin{aligned}
\sum_{q \geq q_{0}} \sup _{2 \leq t \leq T}\langle t\rangle^{\alpha} \int_{1}^{t} e^{c_{0}(\tau-t)} 2^{(N / 2-1) q} S_{q}^{2}(\tau) d \tau & \\
& \lesssim \sum_{q \geq q_{0}} 2^{(N / 2-1) q}\left\|R_{q}\left(\tau v, \tau^{\alpha-1} H^{i j}\right)\right\|_{L_{t}^{\infty}\left(L^{2}\right)} \\
& \lesssim\|\tau \nabla v\|_{\widetilde{L}_{T}^{\infty}\left(\dot{B}_{2,1}^{N / 2}\right)}\left\|\tau^{\alpha-1} H\right\|_{\widetilde{L}_{T}^{\infty}\left(\dot{B}_{2,1}^{N / 2-1}\right)^{2}}
\end{aligned}
$$

The first term on the right side may be bounded thanks to (3.24), and the high frequencies of the last term on the right side are obviously bounded by $D(T)$. As for the low frequencies of the last term, we notice that if $N \leq 6$ 
then for all small enough $\varepsilon>0$,

$$
\begin{aligned}
\left\|\tau^{\alpha-1} H\right\|_{\widetilde{L}_{T}^{\infty}\left(\dot{B}_{2,1}^{N / 2-1}\right)}^{\ell} & \lesssim\left\|\tau^{\alpha-1} H\right\|_{L_{T}^{\infty}\left(\dot{B}_{2,1}^{N / 2-1-2 \varepsilon}\right)}^{\ell} \\
& \lesssim\left\|\tau^{\alpha-1 / 2-N / 2+\varepsilon} \tau^{N / 2-1 / 2-\varepsilon} H\right\|_{L_{T}^{\infty}\left(\dot{B}_{2,1}^{N / 2-1-2 \varepsilon}\right)}^{\ell} \lesssim D(T),
\end{aligned}
$$

and if $N \geq 7$ then

$$
\begin{aligned}
\left\|\tau^{\alpha-1} H\right\|_{\widetilde{L}_{T}^{\infty}\left(\dot{B}_{2,1}^{N / 2-1}\right)}^{\ell} & \lesssim\left\|\tau^{\alpha-1} H\right\|_{L_{T}^{\infty}\left(\dot{B}_{2,1}^{2}\right)}^{\ell} \\
& \lesssim\left\|\tau^{\alpha-N / 4-2} \tau^{N / 4+1} H\right\|_{L_{T}^{\infty}\left(\dot{B}_{2,1}^{2}\right)}^{\ell} \lesssim D(T) .
\end{aligned}
$$

We eventually get

$$
\sum_{q \geq q_{0}} \sup _{2 \leq t \leq T}\langle t\rangle^{\alpha} \int_{1}^{t} e^{c_{0}(\tau-t)} 2^{(N / 2-1) q} S_{q}^{2}(\tau) d \tau \lesssim D(T)^{2} .
$$

Similarly, we have

$$
\sum_{q \geq q_{0}} \sup _{2 \leq t \leq T}\langle t\rangle^{\alpha} \int_{1}^{t} e^{c_{0}(\tau-t)} 2^{(N / 2-1) q} S_{q}^{3}(\tau) d \tau \lesssim D(T)^{2} .
$$

Finally, using product laws, 3.24, 3.28, 3.29) and Lemma 2.9, we obtain

$$
\begin{aligned}
& \sum_{q \geq q_{0}} \sup _{2 \leq t \leq T}\langle t\rangle^{\alpha} \int_{1}^{t} e^{c_{0}(\tau-t)} 2^{(N / 2-1) q} S_{q}^{4}(\tau) d \tau \\
& \quad \lesssim\|\tau \nabla v\|_{\widetilde{L}_{T}^{\infty}\left(\dot{B}_{2,1}^{N / 2}\right)}\left\|\tau^{\alpha-1} \nabla H\right\|_{\widetilde{L}_{T}^{\infty}\left(\dot{B}_{2,1}^{N / 2-1}\right)} \sup _{2 \leq t \leq T} t^{\alpha} \int_{1}^{t} e^{c_{0}(\tau-t)} \tau^{-\alpha} d \tau \\
& \quad \lesssim D(T)^{2}
\end{aligned}
$$

and

$$
\begin{aligned}
& \sum_{q \geq q_{0}} \sup _{2 \leq t \leq T}\langle t\rangle^{\alpha} \int_{1}^{t} e^{c_{0}(\tau-t)} 2^{(N / 2-1) q} S_{q}^{5}(\tau) d \tau \\
& \lesssim\|\tau \nabla v\|_{\widetilde{L}_{T}^{\infty}\left(\dot{B}_{2,1}^{N / 2}\right)}\left\|\tau^{\alpha-1}(\nabla H, d)\right\|_{\widetilde{L}_{T}^{\infty}\left(\dot{B}_{2,1}^{N / 2-1}\right)} \sup _{2 \leq t \leq T} t^{\alpha} \int_{1}^{t} e^{c_{0}(\tau-t)} \tau^{-\alpha} d \tau \\
& \lesssim D(T)^{2}
\end{aligned}
$$

Putting all the above inequalities (3.34)-(3.38) together, we conclude that

$$
\sum_{q \geq q_{0}} \sup _{2 \leq t \leq T}\langle t\rangle^{\alpha} \int_{1}^{t} e^{c_{0}(\tau-t)} 2^{(N / 2-1) q} S_{q}(\tau) d \tau \lesssim X(T)^{2}+D(T)^{2} .
$$

Then plugging (3.22), 3.23) and (3.39) into (3.20) yields

$$
\left\|\langle\tau\rangle^{\alpha}\left(\Lambda H^{i j}, d^{i j}\right)\right\|_{\widetilde{L}_{T}^{\infty}\left(\dot{B}_{2,1}^{N / 2-1}\right)}^{h} \lesssim\left\|\left(\Lambda H_{0}^{i j}, d_{0}^{i j}\right)\right\|_{\dot{B}_{2,1}^{N / 2-1}}^{h}+X(T)^{2}+D(T)^{2} .
$$


STEP 4: Decay estimates with gain of regularity for the high frequencies of $\nabla d^{i j}$. In order to close the estimates of the time-weighted energy functional, this step is devoted to bounding the last terms of $D(t)$. We shall use the fact that the velocity $d^{i j}$ satisfies the equation

$$
\partial_{t} d^{i j}-\mu \Delta d^{i j}=\Lambda H^{i j}+G-v \cdot \nabla d^{i j}=: h .
$$

Therefore,

$$
\partial_{t}\left(t \Delta d^{i j}\right)-\mu \Delta\left(t \Delta d^{i j}\right)=\Delta d^{i j}+t \Delta h .
$$

We deduce from Proposition 2.7 that

$$
\left\|\tau \Delta d^{i j}\right\|_{\widetilde{L}_{t}^{\infty}\left(\dot{B}_{2,1}^{N / 2-1}\right)}^{h} \lesssim\left\|\Delta d^{i j}\right\|_{L_{t}^{1}\left(\dot{B}_{2,1}^{N / 2-1}\right)}^{h}+\|\tau \Delta h\|_{\widetilde{L}_{t}^{\infty}\left(\dot{B}_{2,1}^{N / 2-3}\right)}^{h},
$$

whence, using the bounds given by Theorem 1.1 .

$$
\left\|\tau \nabla d^{i j}\right\|_{\widetilde{L}_{t}^{\infty}\left(\dot{B}_{2,1}^{N / 2}\right)}^{h} \lesssim X(0)+\|\tau h\|_{\widetilde{L}_{t}^{\infty}\left(\dot{B}_{2,1}^{N / 2-1}\right)}^{h} .
$$

In order to bound the first term of $h$, we notice that, because $\alpha \geq 1$ and according to 3.40 , we have

$$
\left\|\tau \Lambda H^{i j}\right\|_{\widetilde{L}_{t}^{\infty}\left(\dot{B}_{2,1}^{N / 2-1}\right)}^{h} \lesssim\left\|\langle\tau\rangle^{\alpha} \Lambda H^{i j}\right\|_{\widetilde{L}_{t}^{\infty}\left(\dot{B}_{2,1}^{N / 2-1}\right)}^{h} \lesssim X(0)+X(t)^{2}+D(t)^{2} .
$$

Noticing that $G=v \cdot \nabla\left(-\Lambda^{-1} \nabla_{j} v^{i}\right)+\Lambda^{-1} \nabla_{j} \mathcal{P}\left(v \cdot \nabla v^{i}-H^{l k} \nabla_{l} H^{i k}\right)+$ $\Lambda^{-1} \nabla_{k}\left(H^{l j} \nabla_{l} H^{i k}-H^{l k} \nabla_{l} H^{i j}\right)$, from (3.24), (3.33) and the definition of $X(t)$ we have

$$
\begin{aligned}
\left\|\tau\left(v \cdot \nabla\left(-\Lambda^{-1} \nabla_{j} v^{i}\right)+\Lambda^{-1} \nabla_{j} \mathcal{P}\left(v \cdot \nabla v^{i}\right)\right)\right\|_{\widetilde{L}_{t}^{\infty}\left(\dot{B}_{2,1}^{N / 2-1}\right)}^{h} & \\
& \lesssim\|v\|_{\widetilde{L}_{t}^{\infty}\left(\dot{B}_{2,1}^{N / 2-1}\right)}\|\tau \nabla v\|_{\widetilde{L}_{t}^{\infty}\left(\dot{B}_{2,1}^{N / 2}\right)} \lesssim X(t) D(t)
\end{aligned}
$$

and

$$
\begin{aligned}
\left\|\tau\left(\Lambda^{-1} \nabla_{j} \mathcal{P}\left(H^{l k} \nabla_{l} H^{i k}\right)+\Lambda^{-1} \nabla_{k}\left(H^{l j} \nabla_{l} H^{i k}-H^{l k} \nabla_{l} H^{i j}\right)\right)\right\|_{\widetilde{L}_{t}^{\infty}\left(\dot{B}_{2,1}^{N / 2-1}\right)}^{h} \\
\lesssim\|\tau H \cdot \nabla H\|_{\widetilde{L}_{t}^{\infty}\left(\dot{B}_{2,1}^{N / 2-1}\right)} \lesssim\left\|\tau^{1 / 2} H\right\|_{\widetilde{L}_{t}^{\infty}\left(\dot{B}_{2,1}^{N / 2}\right)}^{2} \lesssim D(t)^{2} .
\end{aligned}
$$

The third term of $h$ is similar to 3.43 , and we obtain

$$
\left\|\tau\left(v \cdot \nabla d^{i j}\right)\right\|_{\widetilde{L}_{t}^{\infty}\left(\dot{B}_{2,1}^{N / 2-1}\right)}^{h} \lesssim X(t) D(t) .
$$

Hence, reverting to 3.42 , we get

$$
\left\|\tau \nabla d^{i j}\right\|_{\widetilde{L}_{t}^{\infty}\left(\dot{B}_{2,1}^{N / 2}\right)}^{h} \lesssim X(0)+X(t)^{2}+D(t)^{2} .
$$


Finally, adding the resulting inequality to 3.13 and 3.40 yields, for all $t \geq 0$,

$$
\begin{aligned}
D(t) & \lesssim X(0)+D_{0}+\left\|\left(\Lambda H_{0}^{i j}, d_{0}^{i j}\right)\right\|_{\dot{B}_{2,1}^{N / 2-1}}^{h}+X(t)^{2}+D(t)^{2} \\
& \lesssim D_{0}+\left\|\left(\Lambda H_{0}^{i j}, d_{0}^{i j}\right)\right\|_{\dot{B}_{2,1}^{N / 2-1}}^{h}+X(t)^{2}+D(t)^{2},
\end{aligned}
$$

where we have used $X(0)^{\ell}=\left\|\left(H_{0}, v_{0}\right)\right\|_{\dot{B}_{2,1}^{N / 2-1}}^{\ell} \lesssim\left\|\left(H_{0}, v_{0}\right)\right\|_{\dot{B}_{2, \infty}^{-N / 2}}^{\ell}$. As Theorem 1.1 ensures that $X(t)$ is small, one can conclude that $(1.8)$ is fulfilled for all time if $D_{0}$ and $\left\|\left(\Lambda H_{0}^{i j}, d_{0}^{i j}\right)\right\|_{\dot{B}_{2,1}^{N / 2-1}}^{h}$ are small enough. This completes the proof of Theorem 1.2

Acknowledgements. This research was supported by the National Natural Science Foundation of China (11501332, 11771043, 51976112), the Natural Science Foundation of Shandong Province (ZR2015AL007), and Young Scholars Research Fund of Shandong University of Technology.

\section{References}

[1] H. Bahouri, J.-Y. Chemin and R. Danchin, Fourier Analysis and Nonlinear Partial Differential Equations, Grundlehren Math. Wiss. 343, Springer, Heidelberg, 2011.

[2] J.-Y. Chemin, Perfect Incompressible Fluids, Oxford Univ. Press, New York, 1998.

[3] Q. Chen, C. Miao and Z. Zhang, Global well-posedness for compressible Navier-Stokes equations with highly oscillating initial velocity, Comm. Pure Appl. Math. 63 (2010), $1173-1224$.

[4] Y. Chen and P. Zhang, The global existence of small solutions to the incompressible viscoelastic fluid system in 2 and 3 space dimensions, Comm. Partial Differential Equations 31 (2006), 1793-1810.

[5] R. Danchin, Fourier analysis methods for the compressible Navier-Stokes equations, in: Handbook of Mathematical Analysis in Mechanics of Viscous Fluids, Springer, 2018, 1843-1903.

[6] R. Danchin, Well-posedness in critical spaces for barotropic viscous fluids with truly nonconstant density, Comm. Partial Differential Equations 32 (2007), 1373-1397.

[7] R. Danchin, Global existence in critical spaces for compressible Navier-Stokes equations, Invent. Math. 141 (2000), 579-614.

[8] R. Danchin, Density-dependent incompressible viscous fluids in critical spaces, Proc. Roy. Soc. Edinburgh Sect. A 133 (2003), 1311-1334.

[9] M. Gurtin, An Introduction to Continuum Mechanics, Math. Sci. Engrg. 158, Academic Press, 1981.

[10] X. Hu and $\mathrm{H}$. Wu, Long-time behavior and weak-strong uniqueness for incompressible viscoelastic flows, Discrete Contin. Dynam. Systems 35 (2015), 3437-3461.

[11] G. Larson, The Structure and Rheology of Complex Fluids, Oxford Univ. Press, New York, 1998.

[12] Z. Lei, C. Liu and Y. Zhou, Global solutions of incompressible viscoelastic fluids, Arch. Ration. Mech. Anal. 188 (2008), 317-398. 
[13] T. Kobayashi and Y. Shibata, Decay estimates of solutions for the equations of motion of compressible viscous and heat-conductive gases in an exterior domain in $\mathbb{R}^{3}$, Comm. Math. Phys. 200 (1999), 621-659.

[14] A. Matsumura and T. Nishida, The initial value problems for the equations of motion of viscous and heat-conductive gases, J. Math. Kyoto Univ. 20 (1980), 67-104.

[15] J. Qian, Well-posedness in critical spaces for incompressible viscoelastic fluid system, Nonlinear Anal. 72 (2010), 3222-3234.

[16] T. Zhang, Global existence in critical spaces for incompressible viscoelastic fluids, arXiv:1101.5862v1 (2011).

Dandan Ding, Meiling Chi, Fuyi Xu (corresponding auhor)

School of Mathematics and Statistics

Shandong University of Technology

Shandong, China

E-mail: dingdandan217825@163.com

chimeiling0@163.com

zbxufuyi@163.com 
